\title{
Phase Separation of an Evaporating Ternary Solution in a Hele-Shaw Cell
}

\author{
Ricardo Arturo Lopez de la Cruz, Noor Schilder, Xuehua Zhang,* and Detlef Lohse* \\ Cite This: Langmuir 2021, 37, 10450-10460 \\ Read Online
}

ABSTRACT: In the present work, we investigate the dynamic phenomena induced by solvent evaporation from ternary solutions confined in a Hele-Shaw cell. The model solutions consist of ethanol, water, and oil, and with the decrease in ethanol concentration by selective evaporation, they may undergo microdroplet formation via the ouzo effect or macroscopic liquid-liquid phase separation. We varied the initial concentration of the three components of the solutions. For all ternary solutions, evaporation of the good solvent ethanol from the gas-liquid interface, aligned with one side of the cell, leads to a Marangoni instability at the early stage of the evaporation process. The presence of the Marangoni instability is

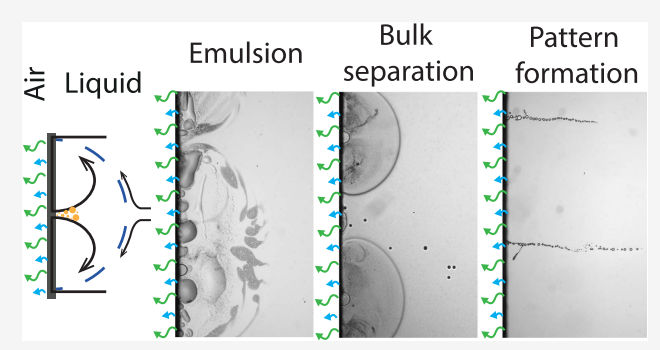
in agreement with our recent predictions based on linear stability analysis of binary systems. However, the location and onset of subsequent microdroplet formation and phase separation are the result of the interplay between the Marangoni instability and the initial composition of the ternary mixtures. We classified the ternary solutions into different groups according to the initial concentration of oil. For each group, based on the ternary diagram of the mixture, we offer a rationale for the way phase separation takes place and discuss how the instability influences droplet nucleation. Our work helps us to understand under what conditions and where droplet nucleation can take place when advection is present during phase separation inside a microfluidic device.

\section{INTRODUCTION}

Evaporation from multicomponent liquid mixtures is ubiquitous in fundamental and applied processes, ${ }^{1}$ such as inkjet printing, ${ }^{2,3}$ microencapsulation by spray drying, ${ }^{4,5}$ concentration of an analyte in chemical diagnosis, ${ }^{6}$ extraction and purification of pharmaceutical ingredients from solutions, ${ }^{7}$ and fabrication of functional materials by droplet templating methods. ${ }^{8-13}$ For example, recent work demonstrates the potential application of evaporating multicomponent droplets in the fabrication of supraparticles. ${ }^{14,15}$ Briefly explained, oil formed from liquidliquid phase separation creates a ring around the drop boundary and acts as a self-lubricating layer to overcome the coffee ring effect. As the evaporating droplets shrink with a freely moving boundary, nanoparticles dispersed inside the drop get assembled into supraparticles of different shapes. ${ }^{14,15}$

Following the pioneering works of Sternling and Scriven ${ }^{16}$ and Linde et al., ${ }^{17}$ our recent work from Lopez de la Cruz et al. ${ }^{18}$ showed that evaporation of a binary solution from an open side of a Hele-Shaw cell can trigger complicated flow dynamics, in particular, a Marangoni instability. In this case, selective evaporation creates a gradient in concentration normal to the gas-liquid interface. If the concentration field is perturbed, concentration gradients along the interface can appear, ultimately leading to the Marangoni instability. It has been shown that enhanced mixing, fast mass transfer, ${ }^{19,20}$ or a fast propagation of chemical fronts ${ }^{21}$ may occur when a Marangoni instability is present at an interface.
Evaporation can also result in the spontaneous emulsification of ternary solutions consisting of ethanol, water, and anise oil, namely, by the so-called ouzo effect. Here, the ouzo effect refers to the spontaneous microdroplet formation in ternary liquid mixtures with compositions between the spinodal and the binodal curves in the solubility phase diagram. ${ }^{22-24}$ These ternary solutions are the favored model systems for understanding evaporation of multicomponent mixtures coupled with liquid-liquid phase separation. ${ }^{1,25,26}$ In general, depending on the composition in the ternary solution, liquid-liquid phase separation can take two routes. It may form two macroscopic subphases via spinodal decomposition ${ }^{27,28}$ or form microdroplets via the ouzo effect, ${ }^{22,29}$ with the relative decrease in the concentration of the good solvent. When confined in a twodimensional (2D) microchamber, surface microdroplets nucleated due to the ouzo effect can self-arrange into intriguing branch-like patterns with a characteristic angle between the merging branches. ${ }^{30}$ Such patterns are a signature of diffusiondominated nucleation and growth of droplets confined in a $2 \mathrm{D}$ space, with a mechanism similar to the ramification of

Received: May 12, 2021

Revised: August 10, 2021

Published: August 23, 2021 
underground rivers over a much larger length scale. ${ }^{31,32}$ Furthermore, the sharp gradient of chemical concentration caused by the phase separation can result in the autonomous motion of the microdroplets due to diffusiophoresis. ${ }^{33,34}$

In the papers mentioned above, ${ }^{30,33,34}$ phase separation of the ternary mixtures was triggered by diffusive mixing with a poor solvent supplied from a deep side channel adjacent to the main $2 \mathrm{D}$ chamber. No gas-liquid interface was responsible for the phase separation or for droplet formation in the chamber. To the best of our knowledge, the study of phase separation induced by evaporation of a ternary mixture confined in a $2 \mathrm{D}$ space remains scant. The fundamental questions to be answered are how does the interfacial tension gradient caused by solvent evaporation affect the mass transfer and liquid-liquid phase separation confined in the $2 \mathrm{D}$ chamber? How is the evaporation-induced behavior correlated with the composition of the solution?

To address those questions, in the present work, we will follow the evaporation of ternary mixtures from a Hele-Shaw cell. As the good solvent evaporates from an opening at the side of the cell, we found that regardless of the initial composition of the ternary solution, a Marangoni instability occurs at the early stage of evaporation, with a mechanism similar to that in the evaporation of binary solutions. However, with time, the processes of mass transfer in the liquid mixture and the formation and evolution of droplets from phase separation display distinct features according to the composition of the initial ternary solution. The oil-rich liquid from phase separation can self-seal the opening of the cell and slow down evaporation. The notable droplet patterns suggest that after self-sealing all subsequent processes become dominated by diffusion.

The organization of the paper is as follows: in the next section, we will present the system and materials used. Afterward, we discuss the Marangoni instability, its evolution in time, and the conditions under which it is triggered. The following four sections are dedicated to describing the four groups in which the mixtures were classified according to the way they phaseseparate. A rationale for the different ways of phase separation is given in each section. We close with a summary and conclusions.

\section{EXPERIMENTAL SECTION}

Chemicals and Solutions. The ternary mixtures in our experiments were made out of water (produced by a reference A+ system, Merck Millipore, at $18.2 \mathrm{M} \Omega \mathrm{cm}$ and $25^{\circ} \mathrm{C}$ ), ethanol (Boom, $100 \%$ (v/ v), technical grade), and trans-anethole (tA, Sigma-Aldrich, 99\%). Initial mass fraction $c_{\alpha}$ of the different mixtures is listed in Table 1, where $\alpha$ refers to ethanol (e), water (w), and trans-anethole (tA). For solutions of the same mass fraction, the error is of $0.1 \mathrm{wt} \%$ as prepared for different runs of experiments. Figure la shows the same mass fractions in a ternary solubility phase diagram. The mixtures were classified into four groups: low (blue), intermediate-low (red), intermediate-high (yellow), and high (green) oil concentrations (see Figure 1a), according to the characteristics of the phase separation.

Configuration of the Hele-Shaw Cell for Evaporation. A microfluidic chip (Beijing First Mems Co., Ltd.) was used as a HeleShaw cell in all of our experiments. Figure $1 \mathrm{~b}$ shows a cross section of the chip. The gap between the two plates was $20 \mu \mathrm{m}$, so the space inside the cell can be considered as quasi-2D. As shown in Figure 1b, a set of micropillars of $50 \mu \mathrm{m}$ in diameter (thin vertical lines in the sketch) and intermediate walls of $0.5 \mathrm{~mm}$ width (thick vertical lines in the sketch) were placed inside the cell to stabilize the top and bottom plates during the fabrication process.

The chips were sealed, except for one side left open to allow for evaporation (left side in Figure 1c,d). Along the opposite side of the chip, there was a subchannel with $50 \mu \mathrm{m}$ in depth, acting as a reservoir to keep the chip filled with the solution (see Figure 1c,d). The surface of
Table 1. Initial Mass Fraction $c_{\alpha}$ of the Different Mixtures Used in Experiments ${ }^{a}$

$\begin{array}{cccc}\text { tag } & c_{\mathrm{e}} \times 100 \% & c_{\mathrm{w}} \times 100 \% & c_{\mathrm{tA}} \times 100 \% \\ \mathrm{a} & 45.8 & 53.4 & 0.8 \\ \mathrm{~b} & 54.9 & 43.1 & 2.0 \\ \mathrm{c} & 60.7 & 35.2 & 4.1 \\ \mathrm{~d} & 63.7 & 30.0 & 6.3 \\ \mathrm{e} & 66.1 & 21.9 & 12.0 \\ \mathrm{f} & 66.5 & 16.0 & 17.5 \\ \mathrm{~g} & 60.0 & 10.0 & 30.0 \\ \mathrm{~h} & 51.1 & 7.3 & 41.6 \\ \mathrm{i} & 35.6 & 4.5 & 59.9 \\ \mathrm{j} & 20.0 & 2.0 & 78.0 \\ \mathrm{k} & 14.0 & 1.0 & 85.0\end{array}$

${ }^{a} \alpha$ refers to e, $\mathrm{w}$, and $\mathrm{tA}$, standing for ethanol, water, and transanethole, respectively. The tags are also displayed in the ternary diagram in Figure 1a.

the chip was hydrophobilized by depositing a layer of trichloro(octadecyl)silane (OTS, Sigma-Aldrich) by a procedure of solution deposition. ${ }^{36}$ In brief, the chip was cleaned in a bath of piranha solution (30\% of hydrogen peroxide and $70 \%$ of sulfuric acid) (caution: piranha solution is highly corrosive and acidic!) for $40 \mathrm{~min}$ at $75{ }^{\circ} \mathrm{C}$. The chip was then taken out and rinsed with plenty of water (Mill-Q $18.2 \mathrm{~m} \Omega$ ) and then with ethanol. The chip was then kept in an oven at $120^{\circ} \mathrm{C}$ for 2 $\mathrm{h}$ to eliminate excessive water on the surface. After cooling down, the chip was soaked in a solution of $0.5 \%$ OTS in toluene for $17 \mathrm{~h}$. Finally, the chip was sonicated for $10 \mathrm{~min}$ in chloroform and $15 \mathrm{~min}$ in toluene, acetone, isopropyl alcohol, ethanol, and water sequentially. After each experiment, the chip was cleaned in a bath of acetone, isopropyl alcohol, and ethanol sequentially. In between each bath, the chip was dried with a flow of nitrogen.

Experimental Procedure and Evaporation Conditions. We followed the evaporation process in a region near the open edge as depicted in Figure 1c by using an inverted microscope (Olympus GX51) with a $5 \times$ objective (Olympus, MPlan FL N, $5 \times / 0.15$ ) in line with a $0.5 \times$ lens for observation and recorded with a charge-coupled device (CCD) camera (MD061MU-SY, Ximea). A complementary metal oxide semiconductor (CMOS) camera (MQ013MG-ON, Ximea) was also used in several experiments. To stitch images, we used a plugin in Image J developed by Preibisch et al. ${ }^{37}$

Fluorescent images were taken with a Nikon confocal microscope A1 system. Perylene (Sigma-Aldrich, $\geq 99 \%$ ) was used to dye two of the oilrich solutions. For mixture h, the mass fraction of perylene was $c_{\mathrm{p}}=4 \times$ $10^{-4}$. For mixture $j$, we had $c_{\mathrm{p}}=5 \times 10^{-5}$. Experiments with the confocal microscope were made in laboratory conditions without relative humidity control. To visualize the flow of some of the mixtures, the solution was doped with tracer particles (FluoSpheres, Life Technologies, $1 \mu \mathrm{m}$ in diameter carboxylate-modified, $2 \%$ solid in aqueous solution with $2 \mathrm{mM}$ of sodium azide).

During the experiments, the solution was delivered from a syringe connected through a capillary tube to the chip. The flow rate was controlled by using a motorized syringe pump (Harvard Instruments, PHD 2000). In each experiment, the chip was filled within $1 \mathrm{~min}$ at a rate of $15 \mu \mathrm{L} / \mathrm{min}$. After $20-30 \mathrm{~s}$, the flow was reduced to a value ranging from 0.005 to $0.5 \mu \mathrm{L} / \mathrm{min}$ to keep the chip filled and compensate for the liquid loss from evaporation. Generally, a solution with a higher oil concentration required a slower rate of the replenishing flow. The direction of the flow is depicted with blue arrows in Figure 1c. Because the gap of the Hele-Shaw cell $(20 \mu \mathrm{m})$ is smaller than the gap of the reservoir channel $(50 \mu \mathrm{m})$, capillary forces keep the cell filled, compensating the evaporated liquid with liquid from the reservoir. To ensure that the capillary pressure was enough to compensate for the evaporation, the outlet was exposed to ambient pressure.

The chip was placed inside a closed environmental chamber where the humidity was controlled by a flow of humid or dry nitrogen. This chamber was created by covering part of the microscope with plastic 


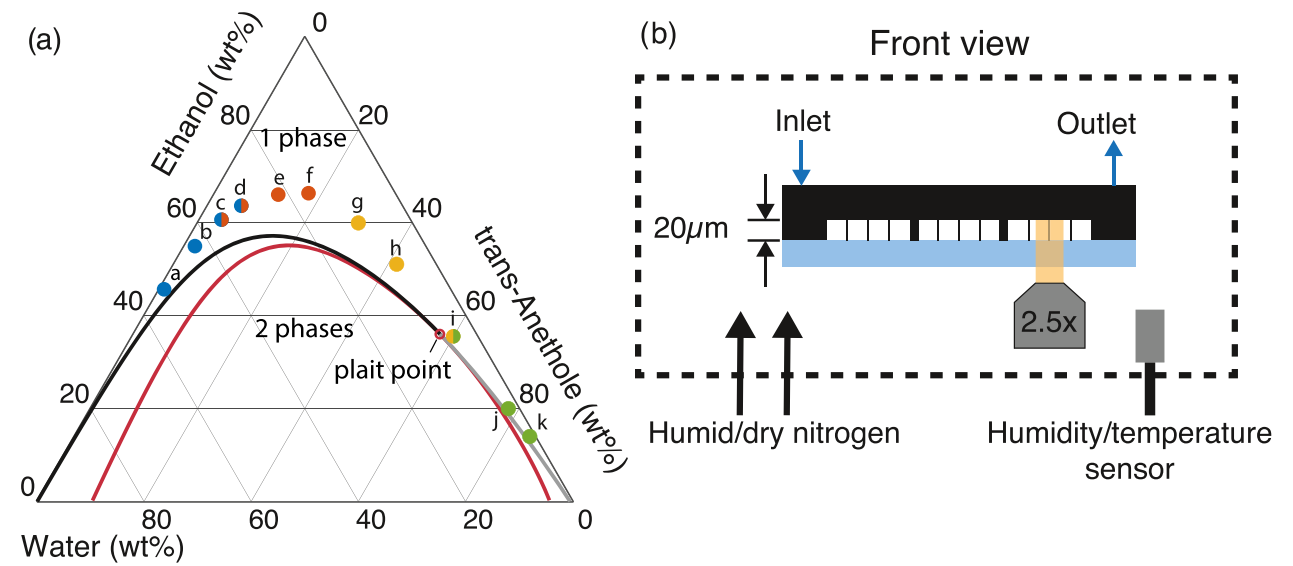

(c)

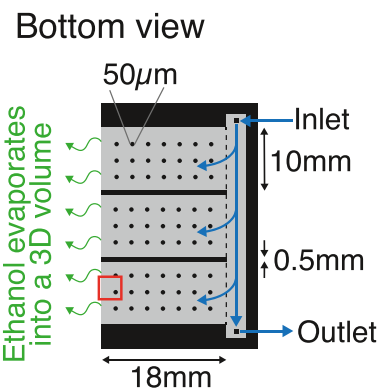

(d)

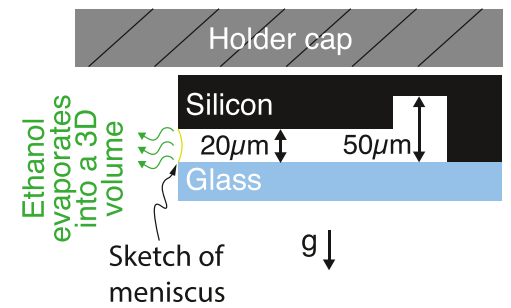

Figure 1. (a) Ternary diagram of ethanol, water, and trans-anethole. The solid lines represent the binodal (black and gray) and the spinodal (red) limits calculated as described in Tan et al. ${ }^{35}$ The initial concentrations used in our experiments are labeled with color-coded circles. Each color represents the solutions that exhibit similar dynamic behavior induced by evaporation and correspond to the same group. Double-colored circles (c, d, and i) are for solutions that have features of two groups. (b) Sketch of the experimental setup showing a frontal view of the Hele-Shaw cell. Black region: silicon frame; light blue region: glass plate for visualization. Thin and thick vertical lines: micropillars and walls to maintain the spacing between the two plates (not to scale). (c) Bottom view of the chip showing the internal structure. Gray areas represent the sections with a gap. The micropillars are depicted as circles and the walls are depicted as horizontal stripes. The two plates are bonded in the black areas. The left edge is open to air, so evaporation can take place. On the right, a channel of $50 \mu \mathrm{m}$ depth was used to fill the chip and keep the volume constant during the experiment. (d) Cross section of the chip with the dimensions of the gap. The position of the meniscus between air and liquid is also sketched.

wrap. The relative humidity was maintained to be within 50 and $52 \%$. When the relative humidity was outside that range, there was a delay before it was back to the desired value, resulting in a larger effective range from approximately $48 \%$ and approximately $53 \%$, depending on the outside humidity. A humidity and temperature sensor (HIH6130, Honeywell) was used to monitor these two quantities. An Arduino board (Arduino Nano) was used to read the signal from the sensor and to control a pair of valves that controlled the flow of humid and dry nitrogen according to the readings of the sensor. The temperature of the gas surrounding the chip was only monitored but not controlled, varying within 20 and $25^{\circ} \mathrm{C}$, for different experiments. Due to the lamp of the microscope, the gas temperature in a single experiment varied approximately at $1-2 \mathrm{~K} / \mathrm{h}$. A figure with the location of the nitrogen inlets and the sensor with respect to the chip are shown in the Supporting Information (SI).

\section{RESULTS AND DISCUSSION}

Evaporation of the most volatile component (ethanol) in the ternary mixture takes place at the gas-liquid interface that was aligned with the open edge of the chip in Figure 1c. In the next section, we will investigate the interesting and complex dynamic phenomena induced by evaporation and the dependence on the initial composition of the ternary solution. The initial solutions were classified into four groups: low, intermediate-low, intermediate-high, and high oil concentrations (see Figure 1a), according to the main characteristics of the phase separation. The differences between groups are not sharp, and the solution labeled with two colors shows characteristics of two adjacent groups.
Initial Marangoni Instability. At the beginning of the evaporation process, a Marangoni instability was triggered for all cases. The instability was evident in the growth and merging of arch-like regions, as shown in Figure 2a. The flow inside the arches is depicted with black arrows and shown with streak lines in Figure $2 \mathrm{~b}$ for the case of $c_{\mathrm{tA}}=0.416$. Such a flow with pairs of convective rolls is typical of Marangoni instabilities. ${ }^{17,18,20,38}$ In our case, the presence of the air-liquid interface and the lower surface tension of volatile ethanol are the origin of the instability, in accordance with previous predictions. ${ }^{16,18}$ For further details in the conditions needed for the instability to be triggered, see the SI.

The horizontal size $x_{\mathrm{F}}$ of the arches increases in time, as shown in Figure 2c. Similarly to binary ethanol-water mixtures, $x_{\mathrm{F}}$ increases approximately as a power law while the instability is active. For low oil contents (blue group), the exponent of the power law is close to $1 / 2$, as in the binary case. ${ }^{18}$ For the rest of the cases, the exponent is larger, up to 0.7 (see the SI). Therefore, it seems that the third component (oil) can help accelerate the arch growth. Furthermore, the lifetime of the Marangoni instability reduces with increasing oil concentration, as shown by the time at which $x_{\mathrm{F}}$ reaches a plateau.

The strong flow at the air-liquid interface makes our evaporating system different from systems where phase separation is induced solely by diffusive mixing. ${ }^{30}$ The flow confines the phase separation inside the arches and prevents surface droplets from self-arranging into branch-like patterns 
(a)

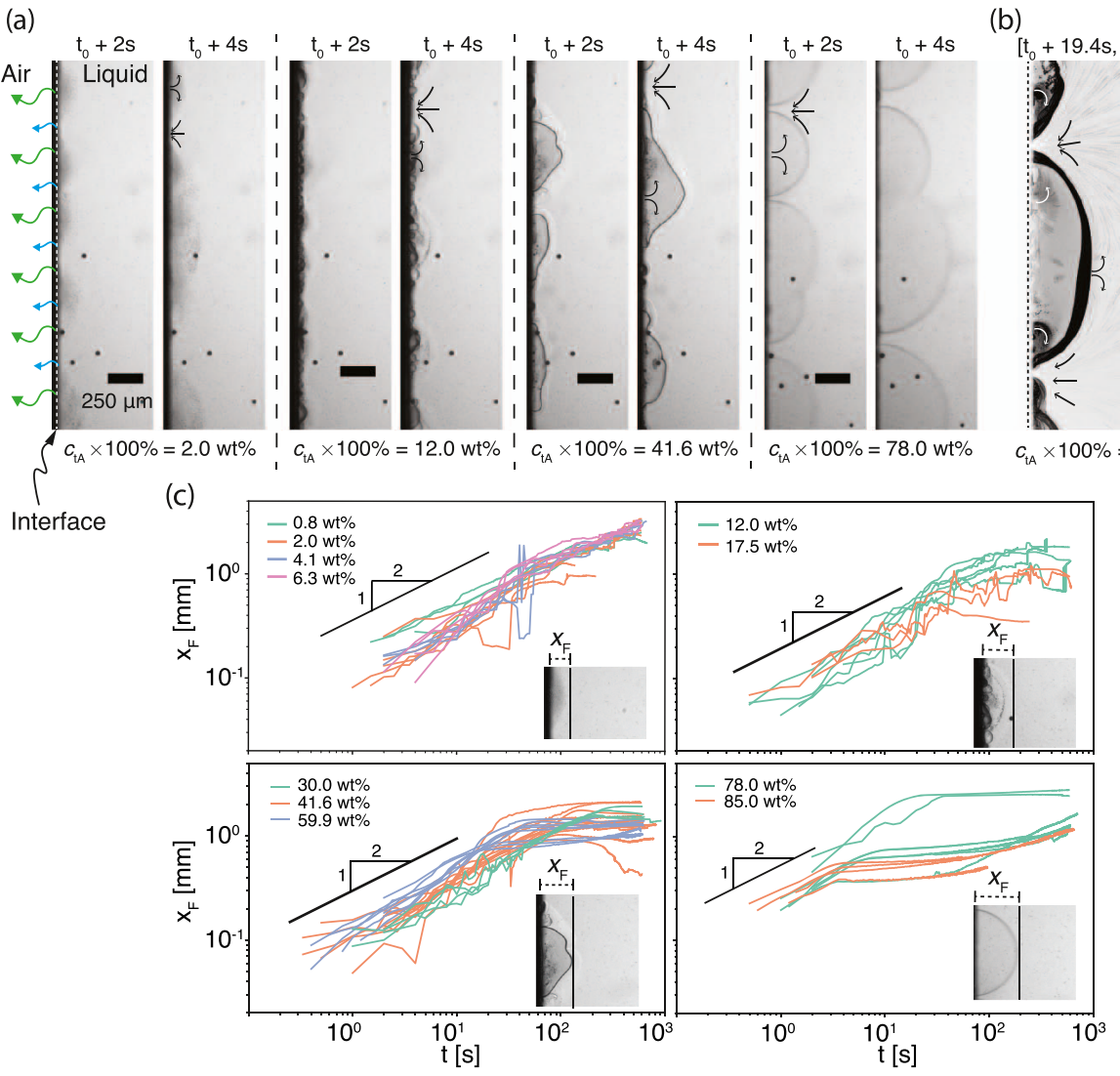

Figure 2. (a) Snapshots showing a section of the Hele-Shaw cell along the open side. The time $t_{0}$ is defined at the moment when the ternary solution covers the edge in the field of view. The initial mass fraction of trans-anethole is labeled below the images. In all cases, phase separation takes place inside arch-like regions. The black arrows indicate the general direction of the flow caused by a Marangoni instability. In the first panel, the vertical white dotted line indicates the location of the air-liquid interface. (b) Composite image obtained from 31 frames for a solution of initial oil mass fraction $c_{\mathrm{tA}}=0.416$ seeded with tracer particles. The arrows indicate the direction of the flow. The vertical black dotted line indicates the position of the air-liquid interface. Movie S1 of the SI corresponds to this figure. (c) Double logarithmic plots showing the distance $x_{\mathrm{F}}$ from the edge of the chip up to the summit of the biggest arch at a given time. The insets in each plot give examples of $x_{\mathrm{F}}$. Each different plot corresponds to one of the four different groups marked in Figure 1a. Curves of the same color represent different repetitions of the same case. For $c_{\mathrm{tA}} \geq 0.120, x_{\mathrm{F}}$ dramatically slows down after the Marangoni instability stops. Lines indicating a $1 / 2$ power law are added for comparison.

close to the interface, in contrast to previous observations. ${ }^{30}$ However, in regions where the flow is slow or after the instability has stopped, diffusion can become dominant and branch-like patterns are recovered. We will discuss in more detail the differences in the spatial distribution of phase separation and droplet nucleation in the following sections.

Group 1: Low Oil Concentration in the Ternary Solution. The solutions in this group are labeled in Figure 3a and also listed in Table 1 as tags $(a-d)$. Ethanol evaporation reduces the concentration of ethanol in the solution while the loss of water and oil is negligible. With progression of evaporation, the ratio of water and oil remains constant before liquid-liquid phase separation and the mixture composition follows the path in the phase diagram. The compositions of all four solutions in this group go through the ouzo region with ethanol loss from evaporation. Therefore, spontaneous emulsification is expected. ${ }^{22,23}$ Indeed, the common features of this group are droplet nucleation and growth. The distribution of the droplets is, in part, associated with the flow caused by the Marangoni instability. Furthermore, away from the gas-liquid interface, groups of surface droplets can arrange themselves into finger-like patterns, as will be described in more detail in the rest of this section. See Figure $3 b$ for examples of the finger-like patterns.
The snapshots in Figure $3 \mathrm{~b}$ show the first 10 min of a typical experiment with $c_{\mathrm{tA}}=0.020$. Initially, oil droplets nucleate from the gas-liquid interface along the open edge of the chip and are distributed inside the arches by the convective rolls, as depicted in the first panels of Figure 3b,c. Other locations of the initial droplet formation within the arches are attributed to the influence of the Marangoni instability on the local composition of the solution. Water-rich solution with less ethanol at the gasliquid interface is transported inside the arches by recirculation, leading to quick depletion of ethanol inside the arches. Therefore, a large concentration gradient normal to the perimeter of the arches (blue dashed line in Figure 3c) builds up, causing the oversaturation of oil and, after tenths of seconds, surface droplet nucleation. The surface droplets at the perimeter of the arches are shown in the second and third panels of Figure $3 \mathrm{~b}$ and sketched in Figure 3c. As the surface droplets become dominant, some of them coalesce into small oil-rich regions that accumulate at the stagnation points between consecutive convective rolls (see Figure $3 \mathrm{~b}, \mathrm{c}$ ).

In parallel to the advective flow caused by the Marangoni instability, the presence of concentration gradients around the droplets can result in diffusiophoretic (noncharged solute) and Marangoni effects at the droplet interface. ${ }^{39,40}$ In some cases, we observed that the surface droplets displace in a direction 


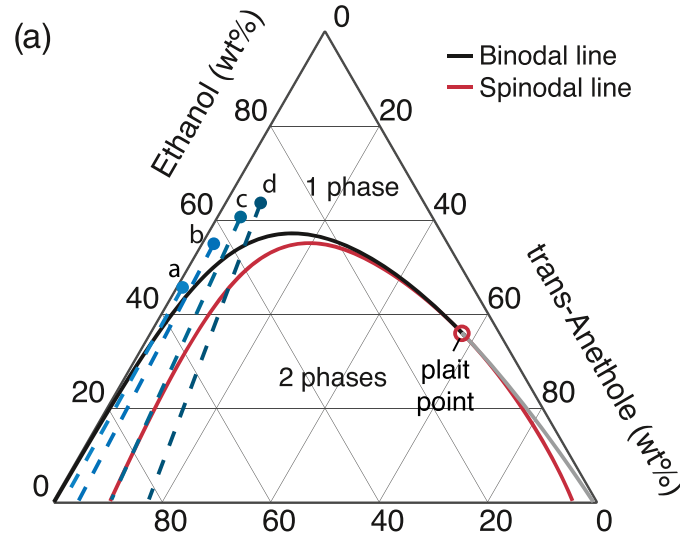

(c)

Water (wt\%)

(b)
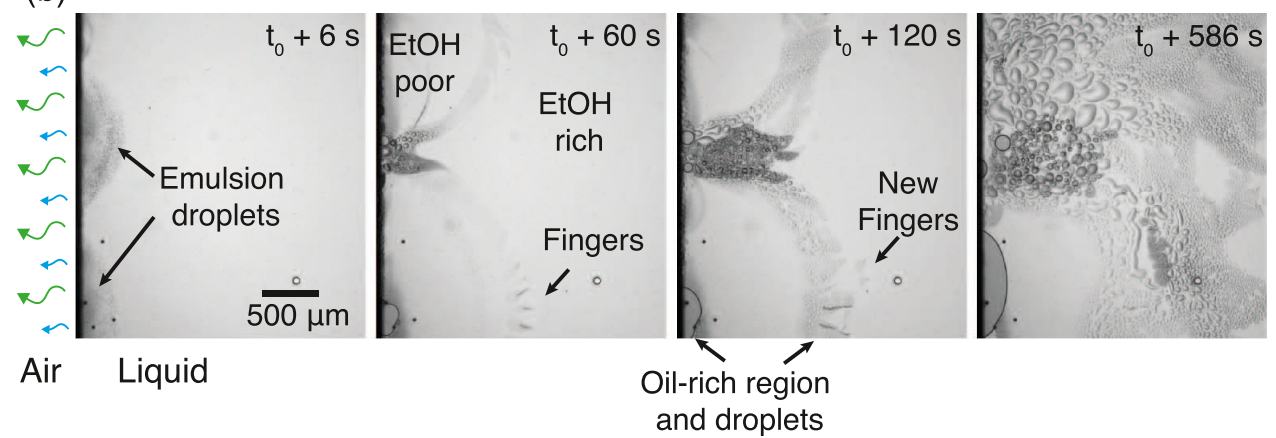

Figure 3. Main characteristics of droplet formation induced by evaporation of ternary solutions with low concentrations of oil $c_{\mathrm{tA}}$. (a) Ternary diagram showing the initial mass fractions in the group. The dashed lines are composition paths for which only ethanol evaporates, while the ratio of water and oil remains constant. (b) Snapshots showing the temporal evolution of a typical evaporation-emulsification process over the first 10 min for an initial concentration ratio $c_{\mathrm{tA}}=0.020$. Movie S2 of the SI corresponds to this case. Evaporation takes place from the left side of each snapshot. (c) Sketch showing the main characteristics observed in the evaporation-emulsification process inside a Marangoni arch over time. The qualitative change in the ethanol concentration $c_{\mathrm{e}}$ and surface tension $\gamma$ is signaled with the superscripts $(++,+,-)$. The solid arrows with open heads indicate the direction of flow. The yellow circles in the left sketch represent bulk droplets. The yellow circles in the right sketch indicate surface droplets, some of which arrange into finger-like patterns.

approximately perpendicular to the perimeter of the arches (see Movie S3). As we already mentioned, we suspect that a strong gradient is present in the direction perpendicular to the perimeter of the arches. Given that both the Marangoni and diffusiophoretic propulsion depend on the existence of a concentration gradient around the droplet, it is possible that both mechanisms are in play here. ${ }^{33}$ However, the direction of displacement can change over time, making it complicated to conclusively determine what is the cause behind droplet displacement once they have nucleated (see Movie S3). Collective effects could also play a role. ${ }^{41}$

In Movie S3, some bulk droplets move in the opposite direction of the surface droplets, possibly indicating the position of the binodal concentration. ${ }^{29}$ However, it is not easy to decouple such a displacement from the advective flow caused by the Marangoni instability or due to the flow caused by displacement of other droplets or larger oil regions. In some cases, we can also observe that bulk droplets or tracer particles flow in circles around surface droplets, further indicating local Marangoni flows. We refer to the work of Zhang et al. ${ }^{33}$ for a closer look into such an interaction between surface and bulk droplets in a ternary system.

Over time, droplet formation inside the cell becomes more and more dominated by diffusion rather than by transport inside the Marangoni arches. It is evident as the droplets self-arrange into finger-like droplet patterns (i.e., signature of diffusion- dominated process ${ }^{30}$ ) at the summit of the arches. The fingers are marked in Figure $3 b$ and sketched in Figure $3 c$ as an elongated group of droplets. The fingers grow approximately in the radial direction with respect to the center of a given arch. However, the finger-like patterns are not long-lived and eventually droplets nucleate in between them (see Movie S2 in the SI), indicating that advection is still present. As the arches become larger, new finger-like patterns appear (third panel of Figure $3 \mathrm{~b}$ ) and get blurred again.

Eventually, the arches become comparable in size with the width of the chip and the surface droplets can nucleate virtually everywhere. In Figure 4, we show two stitched images obtained about $30 \mathrm{~min}$ after the evaporation process started for two different initial concentration ratios. Finger patterns are visible at the frontier (marked with blue dashed lines) between the area with surface droplets and the one-phase area. For the higher oil case, the fingers are more evident and they have ramified into branch-like patterns.

The mechanism for the self-arrangement of the surface droplet in a quasi-2D chamber is explained in the work by Lu et al. ${ }^{30}$ In their case, the emulsification was induced by diffusive mixing with the poor solvent (water), and self-arrangement into branch-like patterns occurred in the entire chamber. In contrast, in our experiments, emulsification is caused by the loss of the good solvent (ethanol) and finger-like patterns only appear locally at the summit of the arches, close to a stagnation point 
(a)

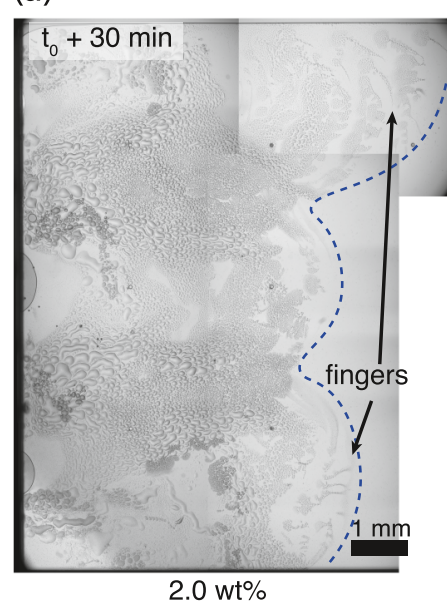

(b)

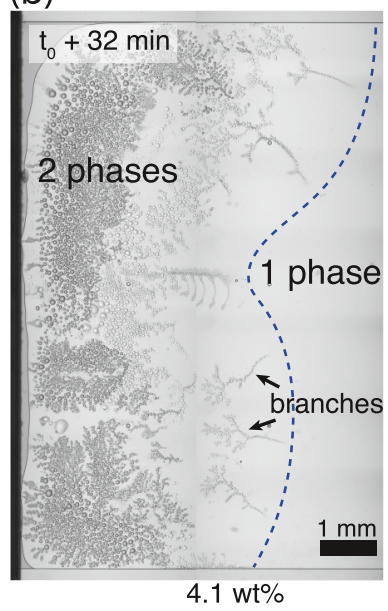

Figure 4. Stitched snapshots showing a larger field of view. In panel (a), $c_{\mathrm{tA}}=0.020$ and the image corresponds to the same series of snapshots shown in Figure $3 \mathrm{~b}$. Finger patterns are visible at the lower-right and upper-right corners. In panel (b), we show an example of $c_{\mathrm{tA}}=0.041$ with ramified fingers. In that case, the snapshots were taken at the middle compartment of the chip (see Figure 1c). In both images, the blue dashed lines qualitatively indicate the frontier between the twophase and one-phase areas considering where oil droplets are visible.

(see Figure 3c). The low velocity close to the stagnation point can cause that, locally, diffusion becomes dominant over advection, thus allowing the formation of the finger-like patterns. Furthermore, for $c_{\mathrm{tA}}=0.041$ and 0.063 , a thin layer of oil can form along the edge. The layer can hinder both the evaporation and the Marangoni flow, supporting our hypothesis that branches and fingers mainly grow due to diffusion.

The formation of the oil layer is in accordance with the composition path of the mixture at $c_{\mathrm{tA}}=0.041$ and 0.063 . For the former, the path passes very close to the spinodal line, and for the latter, the path actually crosses the spinodal limit. We marked mixtures $\mathrm{c}$ and $\mathrm{d}$ with two colors in Figure 1a because a larger accumulation of oil-rich phase is the main feature of the next group.
Group 2: Intermediate-Low Oil Concentration. Figure 5 a shows a ternary diagram with the composition paths corresponding to the group at intermediate-low oil concentration (tags $\mathrm{e}-\mathrm{f}$ in Table 1 ). Since the paths cross a small section of the ouzo region before reaching the spinodal line, we can expect to observe bulk phase separation and areas with noncoalescing droplets simultaneously. Indeed, evaporationinduced behavior for this group of ternary solutions is characterized by a combination of bulk phase separation and emulsification as shown in Figure $5 \mathrm{~b}$ for $c_{\mathrm{tA}}=0.120$.

While the simple straight line evaporation paths are consistent with our observations, a second process might aid in the appearance of noncoalescing droplets. Spinodal decomposition results in two phases with compositions on the binodal line. For the sake of argument, consider compositions $e^{\prime}$ and $e^{\prime \prime}$ plotted in Figure 5a. The latter indicates the composition of the oil-rich agglomerates, and the former gives the composition of the surrounding areas. Since ethanol is continuously evaporating, composition $e^{\prime}$ can enter into a larger portion of the ouzo region, with the corresponding nucleation of new oil droplets. Additionally, notice that $e^{\prime \prime}$ is just above the inverse ouzo region; therefore, the composition of the oil-rich agglomerates can enter the inverse ouzo region as ethanol is lost. From Figure $5 \mathrm{~b}$, we can confirm that, indeed, water-rich droplets form inside the oil-rich regions.

The early stage of phase separation is influenced by Marangoni arches, as shown in Figure 5b. However, this time, the oil droplets that nucleate inside the arches coalesce into large domains of the oil-rich phase with a sharp interface. The oil-rich domains are very mobile, continuously coalescing with each other. Sometimes, the movement is so violent that the oil-rich domains split into two before coalescing again (see Movie S4 in the SI). This behavior is probably a consequence of the nonuniform concentration field around the oil-rich regions, like in the case of droplets inside a nonuniform vapor field. ${ }^{41}$ Both gradients in interfacial tension along the liquid-liquid interface and differences in wetting behavior, together with the hydrophobicity of the walls, could result in motion.

At about $300 \mathrm{~s}$, there are no detectable Marangoni arches anymore and all oil-rich domains have merged into a continuous zone that covers the entire open edge of the cell. It is possible (a)

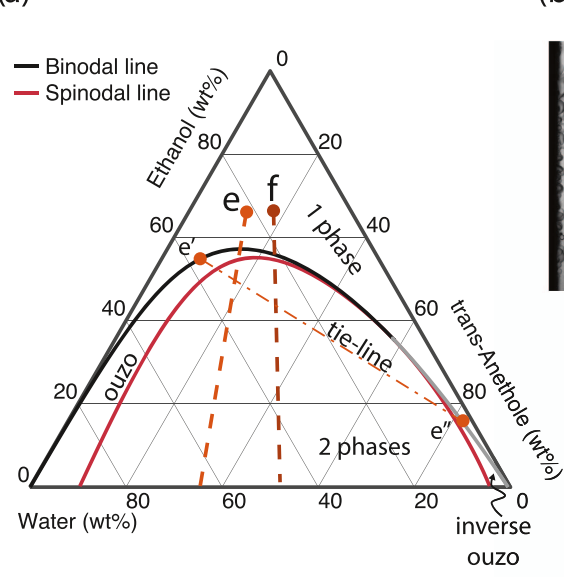

(b)

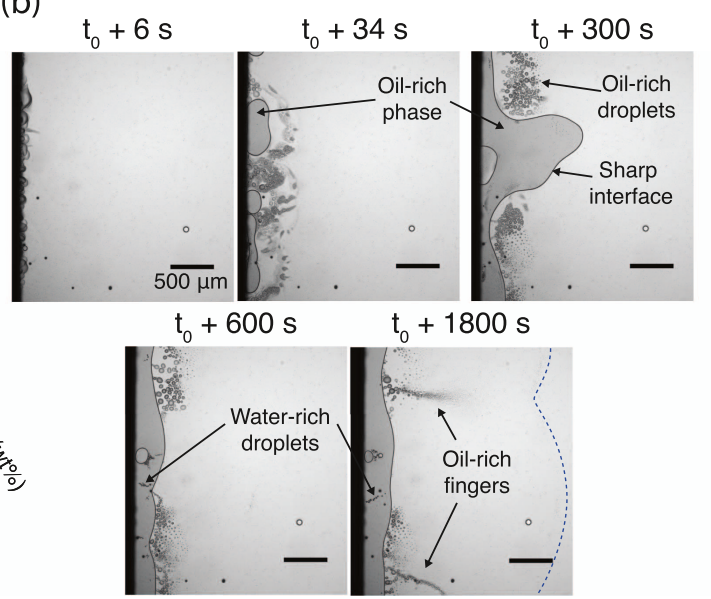

Figure 5. Main characteristics of phase separation induced by evaporation of the solutions with intermediate-low trans-anethole concentrations $c_{\mathrm{tA}}$. (a) Ternary diagram with the initial concentrations corresponding to the red group. The straight dashed lines correspond to processes for which only ethanol evaporates. Points $e^{\prime}$ and $e^{\prime \prime}$ indicate two possible compositions after bulk phase separation. (b) Snapshots at increasing times of a typical example of $c_{\mathrm{tA}}=0.120$. Movie S4 of the SI corresponds to this case. 
(a)

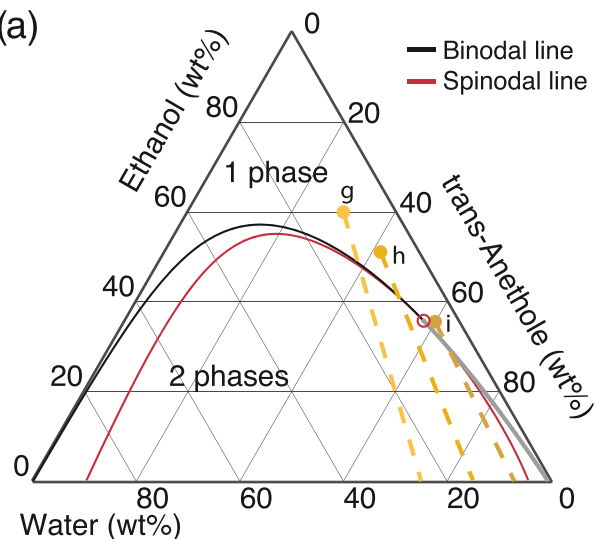

(c)

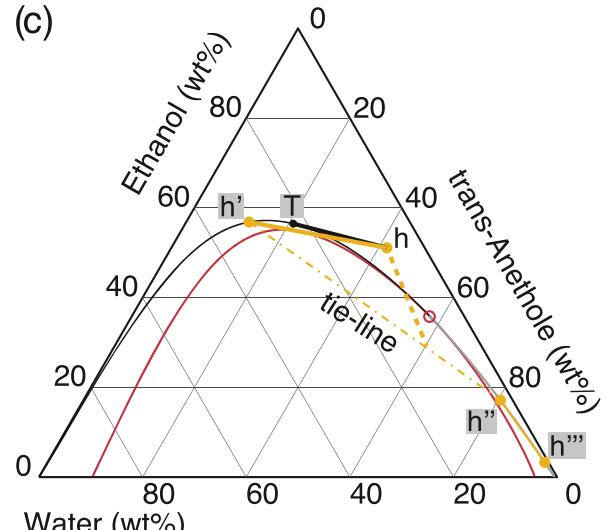

Water (wt\%)

(b)

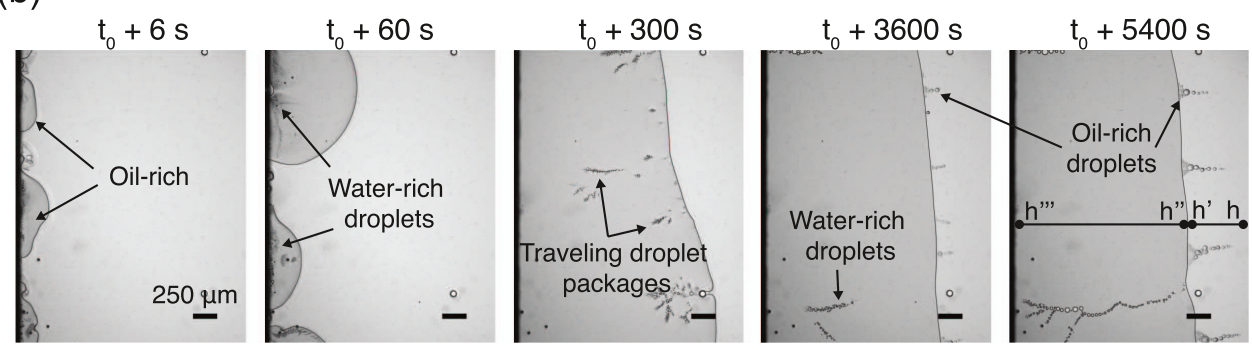

Figure 6. (a) Ternary diagram showing the initial concentrations corresponding to the yellow group (points g-i). The dashed lines correspond to processes for which only ethanol evaporates. (b) Snapshots at increasing times of a typical example of case $h\left(c_{\mathrm{tA}}=0.416\right)$. Movie S5 of the SI corresponds to this case. (c) Ternary diagram with the proposed composition path $h-h^{\prime}-h^{\prime \prime}-h^{\prime \prime \prime}$, corresponding to the black line in the last panel in (b). The dashed line indicates the initial composition path due to evaporation before bulk separation and the dashed-dotted line the tie-line between $h^{\prime}$ and $h^{\prime \prime}$. The black line connecting $h$ and $T$ is tangent to the binodal. In both panels (a) and (c), the open red circle indicates the position of the plait point.

that the continuous oil-rich region reduces the overall surface energy given that a mixture of ethanol and trans-anethole has a lower surface tension than the water-rich solution. Moreover, the interfacial tension between trans-anethole and ethanolwater mixtures is also relatively low (below $25 \mathrm{mN} / \mathrm{m}^{42}$ ). In fact, as shown by the fourth panel in Figure $5 \mathrm{~b}$, the oil-rich region eventually flattens, further reducing the interfacial area, although this could also be an effect of the pressure caused by the replenishing flow. Such a barrier brings the flow to a halt, and evaporation is strongly reduced. Without the Marangoni flow to bring "fresh" solution to the edge, phase separation cannot occur as quickly. However, adjacent to the oil-rich domains, some new droplets can still form and stay on the surface. They are oil-rich droplets, stable in the surrounding water-rich liquid.

Along the boundary of the oil-rich region, droplet fingers form on both sides. Short fingers of water-rich droplets form in the oilrich region, while fingers of oil-rich droplets extend toward the solution in the inner cell (see the last panel of Figure 5b). The fingers of oil-rich droplets ramify with time, extending to the whole width of the cell over approximately $10 \mathrm{~h}$. The emergence of fingers suggests that after the establishment of the oil-rich zone, the mass transfer in the cell becomes dominated by diffusion. ${ }^{30}$ In other words, the solvent evaporation along the opening of the cell is a self-sealing process, creating an oil-rich zone that damps the Marangoni instability, stops moving violently, and reduces the rapid mass loss from the cell. Notice that the slow nucleation of new droplets (to form fingers) indicates that ethanol transport across the oil-rich region and evaporation should continue but at a much slower rate.

Group 3: Intermediate-High Oil Concentration. The mixtures in group 3 are labeled in the ternary diagram shown in Figure $6 \mathrm{a}$ and in Table 1, with tags $\mathrm{g}-\mathrm{i}$. The composition path of the ternary mixtures in this group does not intersect with the ouzo region as the ethanol concentration reduces. Instead, spinodal decomposition is predicted, with the composition of the oil-rich subphase given by a point in the gray side of the binodal line. Moreover, further loss of ethanol can result in the inverse ouzo effect, forming water droplets in the oil-rich phase.

Indeed, the main features induced by evaporation of the mixtures in group 3 are bulk phase separation at early times and the formation of droplet fingers in both the oil-rich and the water-rich regions at late times. As shown in Figure $6 \mathrm{~b}$ for $c_{\mathrm{tA}}=$ 0.416 , large domains of the oil-rich phase have already appeared at time $t=t_{0}+6 \mathrm{~s}$, which is much quicker than in group 2 due to the higher initial concentration of oil in group 3. At $t=t_{0}+300 \mathrm{~s}$, the opening of the cell is fully blocked by the oil-rich phase (Figure 6b). On both sides of the liquid-liquid interface, surface droplets form. Oil droplets nucleate in the water-rich phase from the ouzo effect, and water droplets nucleate in the oil-rich phase from the inverse ouzo effect.

Ethanol evaporation leads to the emergence of a configuration where an oil-rich phase is in contact with a water-rich phase. Ruschak and Miller ${ }^{43}$ studied a similar configuration, where two different mixtures of the same ternary system were put into contact and allowed to interdiffuse. In the cases where the composition path crossed the two-phase region in the ternary diagram, droplet formation was observed, like in our experiments.

To explain our observations of droplet formation, in Figure $6 \mathrm{c}$, we have sketched the possible composition path by following the analysis of Ruschak and Miller. ${ }^{43}$ First, we considered that the mixtures on each side of the liquid-liquid interface are locally in equilibrium. The corresponding compositions are then connected by a tie-line in the ternary diagram. Since water 
(a)

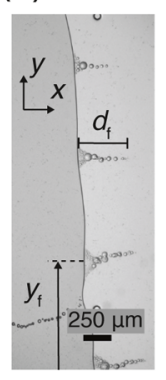

(b)

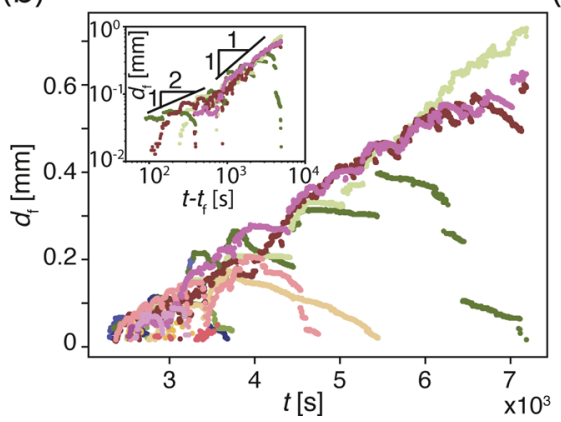

(c)

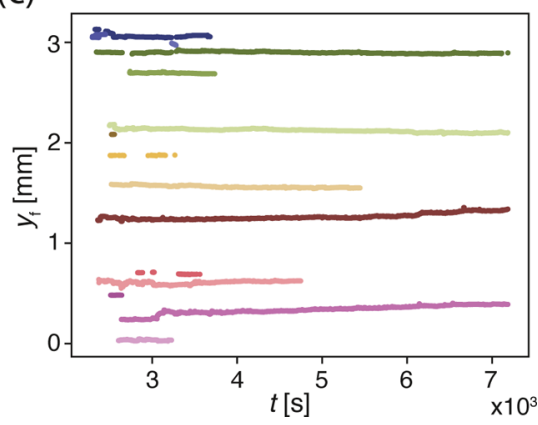

Figure 7. Temporal evolution of the fingers composed of oil-rich droplets. (a) Graphical definition of the quantities used to characterize the fingers. (b) Length $d_{\mathrm{f}}$ of each finger as a function of time. Different colors indicate different fingers. Inset: same data with a double logarithmic scale. Power laws $t^{1 / 2}$ and $t$ are plotted for a comparison with the data to guide the eye. $t_{\mathrm{f}}$ is defined by the moment the first finger appeared. (c) Vertical position $y_{\mathrm{f}}$ of the twodimensional centroid of the fingers as a function of time. The colors correspond with those in panel (b). Time zero in both main plots corresponds to the moment at which the mixture reached the edge of the chip for the first time.

droplets nucleate inside the oil-rich phase, the physical path $h^{\prime \prime \prime}-h^{\prime \prime}$ should cross the inverse ouzo region when mapped into the ternary diagram. While the line $h^{\prime \prime \prime}-h^{\prime \prime}$ is closely parallel to the binodal, it can go inside the inverse ouzo region. On the other side of the interface, the composition should change from an oil-depleted value $h^{\prime}$ at the interface up to the original composition $h$ far away inside the chip. In order for the composition path $h^{\prime}-h$ to cross the two-phase region, point $h^{\prime}$ should lie to the left of the tangency point $\mathrm{T} .{ }^{43}$ While we do not have the exact values of the concentration field and we have assumed straight lines for the composition paths, the constructed path is consistent with nucleation on both sides of the interface. In the SI, we show results with a dyed mixture that seem to support the proposed diffusion path. Nevertheless, further work, possibly in the form of numerical simulations, is needed to confirm our hypothesis.

It is interesting to note that from the liquid-liquid interface water-rich droplets form in "packs". The position of the droplet packs shifts toward the air-liquid interface with time (see Movie S5 in the SI). The way the shift takes place is by formation of new droplets at one end of the pack closer to the air-liquid interface and simultaneous dissolution of the droplets at the other end. Such a shift may be due to the concentration of ethanol always being lower near the air-liquid interface, although the transient nature of the shift (nucleation and dissolution) suggests that there are more mechanisms at play. Some of the packs grow into long-lived branch-like patterns that extend between the liquidliquid and the air-liquid interfaces, as shown in the last snapshot of Figure $6 \mathrm{~b}$. We suspect that the droplet branches are the consequence of the diffusion-dominated mass transfer in the oilrich region, similarly to the fingers formed at the summit of the arches in group 1. Like in group 2, notice that despite the oil-rich phase covering the edge, ethanol transport must continue to take place to trigger phase separation.

The motion of individual water-rich droplets supports the presence of an ethanol concentration gradient in the oil-rich zone. Those droplets move toward the liquid-liquid interface and join the water-rich liquid on the other side of the interface. We suspect that Marangoni flows at the interface of the droplets drive them toward regions of lower surface energy. ${ }^{29,42,44}$ The droplet motion is similar to the self-propulsion of droplets in the work by Zhang et al., ${ }^{33}$ where a concentration gradient is created by liquid-liquid phase separation. On the water-rich side of the liquid-liquid interface, oil-rich droplets appear as shown in the last two snapshots in Figure 6b. Analogously to their water-rich counterparts, some of the oil-rich droplets displace toward the liquid-liquid interface. While moving, the droplets coalesce with those they encounter in their way, leaving the path temporarily free of surface droplets. Later, new droplets nucleate and grow in the freed space. As in group 1, diffusiophoresis ${ }^{40}$ and a nonuniform concentration field, thus nonuniform wetting, ${ }^{41}$ could also play an important role in the displacement of the droplets. For the initial concentration $c_{\mathrm{tA}}=0.598$, the dynamics of the droplets are more complicated and more details are provided in the SI.

Diffusion-Dominated Characteristics of Fingers of Oil-Rich Droplets within a Water-Rich Phase. A number of fingers of oil-rich droplets are shown in the last two panels of Figure 6b. Their temporal evolution is more clearly seen in Figure 7, which shows plots of the length $d_{\mathrm{f}}$ and the vertical position $y_{\mathrm{f}}$ of the fingers over time. A graphical definition of these quantities can be seen in Figure 7a. Figure 7b shows that initially all of the fingers grow, but eventually some shrink and disappear. Therefore, the overall distance between adjacent fingers increases over time, as shown in Figure $7 \mathrm{c}$.

In the inset of Figure $7 \mathrm{~b}$, the length $d_{\mathrm{f}}$ of the droplet fingers that last the longest is plotted on a double logarithmic scale. The time axis is shifted by the moment at which the first finger is visible. There is an indication that initially $d_{\mathrm{f}}$ grows roughly as $\sim t^{1 / 2}$ and then accelerates into a linear regime. The growth mechanism of individual fingers of droplets might be similar to that of the branches observed by Lu et al. ${ }^{30}$ In that study, the process was diffusion-dominated, which can be associated with the initial regime shown in the inset of Figure $7 \mathrm{~b}$.

Lu et al. ${ }^{30}$ concluded that the presence of an already nucleated droplet promoted the formation of new adjacent droplets due to an enhancement in local oversaturation. Moreover, it was observed that adjacent branches grew toward each other, which suggested that there was a long-range interaction between them. We do not observe the formation of branches in this set of experiments (fingers connecting with each other), possibly because of the displacement of the droplets toward the liquidliquid interface. However, the connection of two branches into one, in the work by $\mathrm{Lu}$ et al., ${ }^{30}$ can be seen as a kind of coarsening, as the number of nucleation points decreases. Therefore, the increasing distance between fingers of droplets in our experiments could be the result of a long-range interaction between fingers. Such coarsening can also be seen in the development of fingering instabilities. ${ }^{45-50}$ Additionally, the fingers could compete for oversaturated oil in analogy to the 
(a)

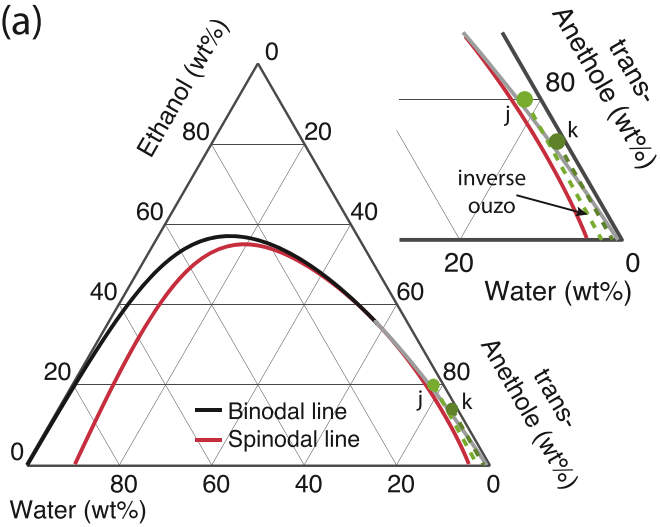

(b)

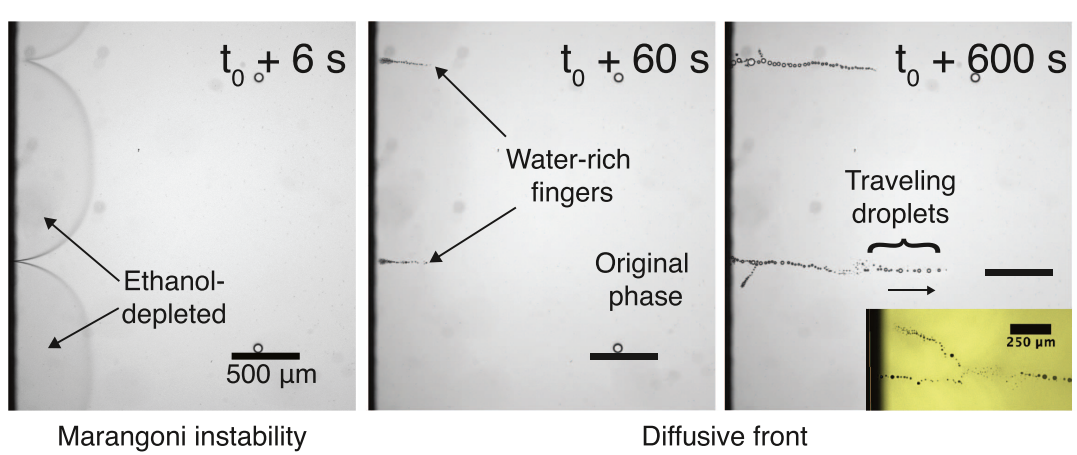

Figure 8. Main characteristics of the evaporation process for high trans-anethole content mixtures. (a) Ternary diagram showing the mixtures corresponding to the green group. The dashed lines correspond to processes for which only ethanol evaporates, while water and oil remain constant. Inset: zoom to the lower-right corner of the diagram. (b) Snapshots at increasing times of a typical example of mixture $j\left(c_{t A}=0.780\right)$. Movie S6 of the SI corresponds to this case. Inset: confocal image of a similar mixture dyed with perylene (nonsoluble in water).

nucleation and growth of droplets in confinement, ${ }^{51}$ the formation of dew, ${ }^{52}$ or in two-dimensional Ostwald ripening. ${ }^{53}$

Finally, the reducing number of fingers might result in more oversaturated oil available per finger, thus favoring a transition to a faster growth. However, an explanation for the change into a linear growth regime must be left for future work.

Group 4: High Oil Concentration. The ternary diagram in Figure 8a shows the initial concentration corresponding to group 4. As before, the evaporation path is plotted, assuming only ethanol loss. Both paths may cross into the inverse ouzo region, which for $c_{\mathrm{tA}}=0.780$ is in accordance with the nucleation of water droplets (Figure $8 \mathrm{~b}$ ). However, on the contrary, for $c_{\mathrm{tA}}=$ 0.850 , there is no phase separation at all. This difference might be explained by the fact that the path is almost parallel to the binodal line. Therefore, the solution may not undergo phase separation.

Snapshots of the first 10 min of the evaporation process for $c_{\mathrm{tA}}$ $=0.780$ are shown in Figure 8b. Initially, the Marangoni arches are the predominant feature, and for a short period of time, some emulsion droplets are visible. For both $c_{\mathrm{tA}}=0.780$ and 0.850 , a diffused interface (slightly darker line in Figure $8 \mathrm{~b}$ ) forms at the perimeter of the arches. However, once the Marangoni instability stops, such an interface is not visible anymore. Instead, the intensity of light changes continuously as shown at $60 \mathrm{~s}$ in Figure 8b. The lack of an interface indicates that the liquid close to the edge is miscible with the bulk liquid.

For $c_{\mathrm{tA}}=0.780$, fingers of water-rich droplets form as indicated in Figure $8 \mathrm{~b}$. We confirmed that the droplets were water-rich by adding a small amount of fluorescent dye that dissolves in ethanol and oil but not in water. The snapshot in the inset of Figure $8 \mathrm{~b}$ shows an example. The fluorescent color (yellow) stays outside of the droplets, indicating that they are water-rich. The length of the fingers increases over time, and they present some ramifications as shown at time $600 \mathrm{~s}$. The ramifications grow from the principal branch, instead of two fingers meeting each other, as mainly observed by Lu et al. ${ }^{30}$

The droplets that compose the fingers increase in size and, eventually, those closer to the tip drift toward the bulk (third panel in Figure 8b and Movie S6 in the SI). While drifting, the droplets dissolve again, indicating that they have reached a highethanol-content environment. Like in the yellow group, the water-rich droplets move up the ethanol gradient in the direction that lowers their surface energy. On their way, coalescence with other growing droplets frees the space behind them. Later on, nucleation and growth take place again in the free space, suggesting that the mixture along the fingers is constantly in the inverse ouzo region.

\section{CONCLUSIONS}

We have investigated dynamical flow phenomena in ternary mixtures of ethanol, water, and trans-anethole confined in a Hele-Shaw cell as evaporation takes place from one side of the cell. Based on the experimental measurements by using optical microscopy, a Marangoni instability occurs at the early stage of the evaporation process, regardless of the initial composition of the solutions. The instability and the induced convective rolls are in accordance with linear stability predictions for binary solutions in two-dimensional ${ }^{16}$ and quasi-two-dimensional ${ }^{18}$ models. Depending on the initial concentration of the ternary mixtures, the duration and the effect of the Marangoni instability on the subsequent liquid-liquid phase separation vary significantly. When droplets have already nucleated, they can be transported by the flow caused by the Marangoni instability, but the nonuniform concentration field can also result in propulsion by a Marangoni flow at the interface of the droplets or by diffusiophoresis. ${ }^{39,40}$ When the initial oil concentration in the ternary mixtures is large, a zone of oil-rich phase forms along the open side of the chip. When the whole edge is covered, the Marangoni instability induced by ethanol evaporation stops. The nucleation of droplets from phase separation and the interactions and evolution of patterns formed by both water-rich and oil-rich droplets in their insoluble surroundings suggest that diffusion-dominated processes take place after the opening is self-sealed by the oil-rich zone.

Our results show that the dynamical behavior induced by evaporation of a ternary system affects how and where phase separation takes place. On the other side, bulk phase separation in combination with the quasi-two-dimensional geometry can eventually hinder advection. Furthermore, the concentration gradient caused by evaporation is coupled with phase separation, too. We find it stunning how rich the flow dynamics in a ternary liquid in such a simple geometry as the Hele-Shaw cell can be. Often beyond our ability to theoretically and quantitatively explain the observed phenomena. However, we hope that nonetheless our study opens paths for further studies, focusing on certain aspects of the different ways in which phase 
separation can take place and its interplay with advection and wetting.

\section{ASSOCIATED CONTENT}

\section{SI Supporting Information}

The Supporting Information is available free of charge at https://pubs.acs.org/doi/10.1021/acs.langmuir.1c01274.

Sketch with the location of the nitrogen inlets and the humidity/temperature sensor; conditions for the appearance of the Marangoni instability and Marangoni number calculation; power law exponents of the growth of $x_{\mathrm{F}}$; confocal images obtained for and example of case $h$ with $c_{\mathrm{tA}}=0.417$; and further details in the phenomena observed for case i, $c_{\mathrm{tA}}=0.599$ (PDF)

Movie corresponding to the seeded case shown in Figure 2b (Movie S1) (MP4)

Movie corresponding to the snapshot of Figure 3 (Movie S2) (MP4)

Movie showing examples of droplet displacement for trans-anethole concentrations of $c_{\mathrm{tA}}=0.041$ and 0.063 (Movie S3) (MP4)

Movie corresponding to the snapshot of Figure 5 (Movie S4) (MP4)

Movie corresponding to the snapshot of Figure 6 (Movie S5) (MP4)

Movie corresponding to the snapshot of Figure 8 (Movie S6) (MP4)

\section{AUTHOR INFORMATION}

\section{Corresponding Authors}

Xuehua Zhang - Physics of Fluids Group, Max-Planck-Center Twente for Complex Fluid Dynamics, Mesa+ Institute, and J. M. Burgers Centre for Fluid Dynamics, Faculty of Science and Technology, University of Twente, 7500 AE Enschede, The Netherlands; Department of Chemical and Materials Engineering, University of Alberta, Edmonton, Alberta T6G 1H9, Canada; (1) orcid.org/0000-0001-6093-5324; Email: xuehua.zhang@ualberta.ca

Detlef Lohse - Physics of Fluids Group, Max-Planck-Center Twente for Complex Fluid Dynamics, Mesa+ Institute, and J. M. Burgers Centre for Fluid Dynamics, Faculty of Science and Technology, University of Twente, 7500 AE Enschede, The Netherlands; Max Planck Institute for Dynamics and SelfOrganization, 37077 Göttingen, Germany; 이이.org/ 0000-0003-4138-2255; Email: d.lohse@utwente.nl

\section{Authors}

Ricardo Arturo Lopez de la Cruz - Physics of Fluids Group, Max-Planck-Center Twente for Complex Fluid Dynamics, Mesa+ Institute, and J. M. Burgers Centre for Fluid Dynamics, Faculty of Science and Technology, University of Twente, 7500 AE Enschede, The Netherlands; (1) orcid.org/0000-00022139-6257

Noor Schilder - Physics of Fluids Group, Max-Planck-Center Twente for Complex Fluid Dynamics, Mesa+ Institute, and J. M. Burgers Centre for Fluid Dynamics, Faculty of Science and Technology, University of Twente, 7500 AE Enschede, The Netherlands

Complete contact information is available at: https://pubs.acs.org/10.1021/acs.langmuir.1c01274

\section{Notes}

The authors declare no competing financial interest.

\section{ACKNOWLEDGMENTS}

The authors thank the insightful conversations with H. Tan, Y. Li, P. Kant, K. Leong Chong, and C. Diddens. The authors thank C. Diddens for sharing the data of the binodal and spinodal lines used in the ternary phase diagrams. The authors acknowledge support from NWO under the ERC-Advanced Grant under Project no. 740479 and from the ERC-Proof-of-Concept Grant under Project no. 862032. X.Z. acknowledges support from the Natural Science and Engineering Council of Canada (NSERC) and from the Canada Research Chairs program.

\section{REFERENCES}

(1) Lohse, D.; Zhang, X. Physicochemical hydrodynamics of droplets out of equilibrium. Nat. Rev. Phys. 2020, 2, 426-443.

(2) Tao, R.; Fang, Z.; Zhang, J.; Ning, H.; Chen, J.; Yang, C.; Zhou, Y.; Yao, R.; Lin, W.; Peng, J. Critical Impact of Solvent Evaporation on the Resolution of Inkjet Printed Nanoparticles Film. ACS Appl. Mater. Interfaces 2018, 10, 22883-22888.

(3) Lohse, D. Fundamental Fluid Dynamics Challenges in Inkjet Printing. Annu. Rev. Fluid Mech., in press 2022, 54.

(4) Ré, M. Microencapsulation by spray drying. Drying Technol. 1998, 16, 1195-1236.

(5) Khelissa, S.; Gharsallaoui, A.; Wang, J.; Dumas, E.; Barras, A.; Jama, C.; Jbilou, F.; Loukili, N.; Chihib, N. Anti-biofilm activity of dodecyltrimethylammonium chloride microcapsules against Salmonella enterica serovar Enteritidis and Staphylococcus aureus. Biofouling 2021, 37, 49-60.

(6) Wong, S. Y.; Cabodi, M.; Rolland, J.; Klapperich, C. M. Evaporative Concentration on a Paper-Based Device to Concentrate Analytes in a Biological Fluid. Anal. Chem. 2014, 86, 11981-11985.

(7) Tchakalova, V.; Zemb, T.; Benczédi, D. Evaporation triggered selfassembly in aqueous fragrance-ethanol mixtures and its impact on fragrance performance. Colloids Surf., A 2014, 460, 414-421.

(8) Zhang, A.; Bai, H.; Li, L. Breath figure: a nature-inspired preparation method for ordered porous films. Chem. Rev. 2015, 115, 9801-9868.

(9) Huang, J.; Zhu, J.; Sun, W.; Ji, J. Versatile and Functional Surface Patterning of in Situ Breath Figure Pore Formation via Solvent Treatment. ACS Appl. Mater. Interfaces 2020, 12, 47048-47058.

(10) Bui, V.; Thuy, L. T.; Tran, Q. C.; Nguyen, V.; Dao, V.; Choi, J. S.; Choi, $\mathrm{H}$. Ordered honeycomb biocompatible polymer films via a onestep solution-immersion phase separation used as a scaffold for cell cultures. Chem. Eng. J. 2017, 320, 561-569.

(11) Cheng, R.; Colombo, R. N. P.; Zhang, L.; Nguyen, D. H. T.; Tilley, R.; Cordoba de Torresi, S. I.; Dai, L.; Gooding, J. J.; Gonçales, V. R. Porous Graphene Oxide Films Prepared via the Breath-Figure Method: A Simple Strategy for Switching Access of Redox Species to an Electrode Surface. ACS Appl. Mater. Interfaces 2020, 12, 55181-55188.

(12) Murchio, S.; Ding, Y.; Speranza, G.; Sorarù, G. D.; Maniglio, D. Ultrasound-Assisted Hydroxyapatite-Decorated Breath-Figure Polymer-Derived Ceramic Coatings for Ti6Al4V Substrates. ACS Appl. Mater. Interfaces 2020, 12, 50772-50783.

(13) Kang, S.; Ryu, D. Y.; Ringe, E.; Hickey, R. J.; Park, S. Nanoparticle-Induced Self-Assembly of Block Copolymers into Nanoporous Films at the Air-Water Interface. ACS Nano 2020, 14, 12203-12209.

(14) Tan, H.; Wooh, S.; Butt, H.-J.; Zhang, X.; Lohse, D. Porous supraparticle assembly through self-lubricating evaporating colloidal ouzo drops. Nat. Commun. 2019, 10, No. 478.

(15) Thayyil Raju, L.; Koshkina, O.; Tan, H.; Riedinger, A.; Landfester, K.; Lohse, D.; Zhang, X. Particle Size Determines the Shape of Supraparticles in Self-Lubricating Ternary Droplets. ACS Nano 2021, 15, 4256-4267. 
(16) Sternling, C. V.; Scriven, L. E. Interfacial Turbulence: Hydrodynamic Instability and the Marangoni Effect. AIChE J. 1959, $5,514-523$.

(17) Linde, H.; Pfaff, S.; Zirkel, C. Strömungsuntersuchungen zur hydrodynamischen Instabilität flüssig-gasförmiger Phasengrenzen mit Hilfe der Kapillarspaltmethode. Z. Phys. Chem. 1964, 225O, 72-100.

(18) Lopez de la Cruz, R. A.; Diddens, C.; Zhang, X.; Lohse, D. Marangoni instability triggered by selective evaporation of a binary liquid inside a Hele-Shaw cell. J. Fluid Mech. 2021, 923, A16.

(19) Grahn, A. Two-dimensional numerical simulations of Marangoni-Bénard instabilities during liquid-liquid mass transfer in a vertical gap. Chem. Eng. Sci. 2006, 61, 3586-3592.

(20) Schwarzenberger, K.; Köllner, T.; Linde, H.; Boeck, T.; Odenbach, S.; Eckert, K. Pattern formation and mass transfer under stationary solutal Marangoni instability. Adv. Colloid Interface Sci. 2014, 206, 344-371.

(21) Shi, Y.; Eckert, K. Acceleration of reaction fronts by hydrodynamic instabilities in immiscible systems. Chem. Eng. Sci. 2006, 61, 5523-5533.

(22) Vitale, S. A.; Katz, J. L. Liquid droplet dispersions formed by homogeneous liquid- liquid nucleation: "The Ouzo effect". Langmuir 2003, 19, 4105-4110.

(23) Sitnikova, N. L.; Sprik, R.; Wegdam, G.; Eiser, E. Spontaneously formed trans-anethol/water/alcohol emulsions: mechanism of formation and stability. Langmuir 2005, 21, 7083-7089.

(24) Grillo, I. Small-angle neutron scattering study of a world-wide known emulsion: Le Pastis. Colloids Surf., A 2003, 225, 153-160.

(25) Tan, H.; Diddens, C.; Lv, P.; Kuerten, J. G. M.; Zhang, X.; Lohse, D. Evaporation-triggered microdroplet nucleation and the four life phases of an evaporating Ouzo drop. Proc. Natl. Acad. Sci. U.S.A. 2016, $113,8642-8647$.

(26) Tan, H.; Diddens, C.; Versluis, M.; Butt, H.; Lohse, D.; Zhang, X. Self-wrapping of an ouzo drop induced by evaporation on a superamphiphobic surface. Soft Matter 2017, 13, 2749-2759.

(27) Moerman, P. G.; Hohenberg, P. C.; Vanden-Eijnden, E.; Brujic, J. Emulsion patterns in the wake of a liquid-liquid phase separation front. Proc. Natl. Acad. Sci. U.S.A. 2018, 115, 3599-3604.

(28) Tree, D. R.; Dos Santos, L. F.; Wilson, C. B.; Scott, T. R.; Garcia, J. U.; Fredrickson, G. H. Mass-transfer driven spinodal decomposition in a ternary polymer solution. Soft Matter 2019, 15, 4614-4628.

(29) Hajian, R.; Hardt, S. Formation and lateral migration of nanodroplets via solvent shifting in a microfluidic device. Microfluid. Nanofluid. 2015, 19, 1281-1296.

(30) Lu, Z.; Schaarsberg, M. H. K.; Zhu, X.; Yeo, L. Y.; Lohse, D.; Zhang, $\mathrm{X}$. Universal nanodroplet branches from confining the Ouzo effect. Proc. Natl. Acad. Sci. U.S.A. 2017, 114, 10332-10337.

(31) Cohen, Y.; Devauchelle, O.; Seybold, H. F.; Robert, S. Y.; Szymczak, P.; Rothman, D. H. Path selection in the growth of rivers. Proc. Natl. Acad. Sci. U.S.A. 2015, 112, 14132-14137.

(32) Devauchelle, O.; Petroff, A. P.; Seybold, H. F.; Rothman, D. H. Ramification of stream networks. Proc. Natl. Acad. Sci. U.S.A. 2012, 109, 20832-20836.

(33) Zhang, X.; You, J. B.; Arends, G. F.; Qian, J.; Chen, Y.; Lohse, D.; Shaw, J. M. Propelling Microdroplets Generated and Sustained by Liquid-liquid Phase Separation in Confined Spaces. Soft Matter 2021, $17,5362-5374$.

(34) Arends, G. F.; You, J. B.; Shaw, J. M.; Zhang, X. Enhanced displacement of phase separating liquid mixtures in $2 \mathrm{D}$ confined spaces. Energy Fuels 2021, 35, 5194-5205.

(35) Tan, H.; Diddens, C.; Mohammed, A. A.; Li, J.; Versluis, M.; Zhang, X.; Lohse, D. Microdroplet nucleation by dissolution of a multicomponent drop in a host liquid. J. Fluid Mech. 2019, 870, 217246.

(36) Zhang, X. H.; Maeda, N.; Craig, V. S. J. Physical Properties of Nanobubbles on Hydrophobic Surfaces in Water and Aqueous Solutions. Langmuir 2006, 22, 5025-5035.

(37) Preibisch, S.; Saalfeld, S.; Tomancak, P. Globally optimal stitching of tiled $3 \mathrm{D}$ microscopic image acquisitions. Bioinformatics 2009, 25, 1463-1465.
(38) Köllner, T.; Schwarzenberger, K.; Eckert, K.; Boeck, T. Solutal Marangoni convection in a Hele-Shaw geometry: Impact of orientation and gap width. Eur. Phys. J.: Spec. Top. 2015, 224, 261-276.

(39) Anderson, J. L. Colloid transport by interfacial forces. Annu. Rev. Fluid Mech. 1989, 21, 61-99.

(40) Ruckenstein, E. Can phoretic motions be treated as interfacial tension gradient driven phenomena? J. Colloid Interface Sci. 1981, 83, $77-81$.

(41) Cira, N. J.; Benusiglio, A.; Prakash, M. Vapour-mediated sensing and motility in two-component droplets. Nature 2015, 519, 446-450.

(42) Li, Y.; Diddens, C.; Prosperetti, A.; Chong, K. L.; Zhang, X.; Lohse, D. Bouncing Oil Droplet in a Stratified Liquid and its Sudden Death. Phys. Rev. Lett. 2019, 122, No. 154502.

(43) Ruschak, K. J.; Miller, C. A. Spontaneous Emulsification in Ternary Systems with Mass Transfer. Ind. Eng. Chem. Fundam. 1972, 11, 534-540.

(44) Gallaire, F.; Meliga, P.; Laure, P.; Baroud, C. N. Marangoni induced force on a drop in a Hele Shaw cell. Phys. Fluids 2014, 26, No. 062105.

(45) Böckmann, M.; Müller, S. C. Coarsening in the buoyancy-driven instability of a reaction-diffusion front. Phys. Rev. E 2004, 70, No. 046302.

(46) De Wit, A. Chemo-hydrodynamic patterns and instabilities. Annu. Rev. Fluid Mech. 2020, 52, 531-555.

(47) Eckert, K.; Acker, M.; Shi, Y. Chemical pattern formation driven by a neutralization reaction. I. Mechanism and basic features. Phys. Fluids 2004, 16, 385-399.

(48) Horváth, D.; Tóth, A. Diffusion-driven front instabilities in the chlorite-tetrathionate reaction. J. Chem. Phys. 1998, 108, 1447-1451.

(49) Huang, J.; Edwards, B. F. Pattern formation and evolution near autocatalytic reaction fronts in a narrow vertical slab. Phys. Rev. E 1996, $54,2620$.

(50) Saffman, P. G.; Taylor, G. I. The penetration of a fluid into a porous medium or Hele-Shaw cell containing a more viscous liquid. Proc. R. Soc. London, Ser. A 1958, 245, 312-329.

(51) Peng, S.; Lohse, D.; Zhang, X. Spontaneous pattern formation of surface nanodroplets from competitive growth. ACS Nano 2015, 9, 11916-11923.

(52) Beysens, D. Dew nucleation and growth. C. R. Phys. 2006, 7, $1082-1100$.

(53) Krichevsky, O.; Stavans, J. Correlated Ostwald ripening in two dimensions. Phys. Rev. Lett. 1993, 70, 1473. 